Historia y comunicación social

ISSN-e 1988-3056

\title{
Efecto de los post en Facebook de los principales candidatos españoles en las elecciones generales de 2016 sobre la polarización de la sociedad
}

\author{
Paloma Abejón Mendoza ${ }^{1}$; Rafael Carrasco Polaino²; Miguel L. Garralón ${ }^{3}$
}

Recibido: 30 de julio de 2018. / Aceptado: 16 de mayo de 2019.

Resumen. Según Hallin y Mancini (2004), el sistema mediático español encaja en el modelo denominado como pluralismo polarizado, que describe una situación en la que los medios de comunicación no actúan como elementos independientes del poder político, sino que se entregan al proselitismo y el ataque al adversario. El objetivo de esta investigación es comprobar cómo se comporta la red social Facebook en relación al fenómeno de la polarización política. Se analizarán cualitativamente los post de los cuatros principales candidatos a la presidencia del Gobierno en las últimas elecciones generales (26 de Junio de 2016) durante una semana de la campaña electoral (del 19 al 25 de junio) y se valorará el nivel de polarización que generaron atendiendo al número de comentarios recibidos.

Palabras clave: polarización; comunicación política; redes sociales.

\section{[en] The effect of the Facebook posts of the main Spanish candidates in the 2016 elections on the polarization of society}

\begin{abstract}
According to Hallin and Mancini (2004), the Spanish media system fits in the Polarized Pluralist Model. This model shows a situation where the media don't act as an independent element of the politic power. Instead of that, the media give themselves up to proselytism and the attack to the opponent. The objective of this investigation is to prove how the social media Facebook works in relationship with the political polarization phenomenon. It will be analysed qualitatively the post of the four leading candidates to the Spanish government in the last general elections (June 26 $6^{\text {th }}, 2016$ ) during one week of the electoral campaign (from the $19^{\text {th }}$ to $25^{\text {th }}$ of June). It will be considered the polarization level generated by the post attending to the number of the sent comments.
\end{abstract}

Keywords: polarization; political communication; social networks.

Sumario: 1. Introducción y estado de la cuestión; 2. Objetivos y metodología; 3. Resultados 4. Discusión y conclusiones. 5. Bibliografía.

Cómo citar: Abejón Mendoza, P.; Carrasco Polaino, R.; Garralón, M. L. (2019). Efecto de los post en Facebook de los principales candidatos españoles en las elecciones generales de 2016 sobre la polarización de la sociedad, en Historia y comunicación social 24 (2), 599-613.

\footnotetext{
$1 \quad$ Universidad Complutense de Madrid. pabejonm@ucm.es

2 Universidad Complutense de Madrid. racarras@ucm.es

$3 \quad$ Universidad Complutense de Madrid. miglop07@ucm.es
} 


\section{Introducción y estado de la cuestión}

La investigación parte de la hipótesis de que la política es hoy más confrontación que conversación, basándonos en las dificultades que hubo para formar un gobierno tras las elecciones de diciembre de 2015 en España y la necesidad de repetir elecciones en junio de 2016.

Se propone analizar si Facebook contribuye o no a mejorar la cultura de la conversación en el ámbito político o si, por el contrario, tiende a polarizar a la sociedad. Y para ello esta investigación llevará a cabo un análisis cualitativo de los post de los principales candidatos durante la semana de campaña del 19 al 25 de junio de 2016. El análisis parte del supuesto de que las técnicas de análisis cualitativo tienen cada vez más importancia en el ámbito de las ciencias sociales y compartimos con Piñuel (2002) que sólo los análisis verificativos y explicativos pueden dar cuenta de inferencias sobre el origen, naturaleza, funcionamiento y efectos de los productos comunicativos.

El análisis llevado a cabo seleccionó los post de los candidatos en el periodo de tiempo elegido que más comentarios recibieron y el objetivo fue detallar qué conjuntos de palabras provocaron ese desencadenante. Tal y como desarrolla Van Dijk (2003) en el marco de su propuesta general de la ideología, la expresión discursiva del cuadrante ideológico se presenta en todos los niveles, es decir, el léxico y semántico; sintáctico, pragmático y retórico. En cada caso, se trata de enfatizar en las cosas positivas de unos y enfatizar en las cosas negativas de los otros.

Martín-Baró (1983) ya determinó que al polarizarse, una persona se identifica con un grupo y asume su forma de captar un problema, lo que le lleva a rechazar la postura opuesta y a las personas que la sostienen. Desde el punto de vista cognoscitivo, la persona polarizada reduce su percepción acerca del grupo rival a estereotipos, a categorías simplistas y rígidas, que contienen una mínima identificación grupal y una fuerte caracterización negativa de orden moral.

La elección de la red social Facebook como objeto de estudio viene determinada porque es la red social más popular y utilizada por más internautas en el mundo, con cerca de 1.5 miles de millones de usuarios activos actualmente (Brandtzaeg, 2015; Woolley et al., 2010). En España, en 2016 había unos 24 millones de usuarios que tenían un perfil activo en esta red social, según el portal de estadísticas Statista, y, además, no hay estudios específicos sobre polarización y redes referentes a esta plataforma y sí referidos a Twitter (Bustos y Capilla, 2013). El único estudio especíico sobre polarización política y Facebook es referido a Colombia (Acosta 2012).

En el marco del análisis ideológico del discurso, Van Dijk (2003) ya sostenía que la polarización es una estrategia discursiva en la que se pone en marcha el mecanismo global de auto-presentación positiva de unos frente a la presentación negativa de los otros.

Hay numerosos estudios sobre cómo la estructura de las redes sociales por donde se difunden las opiniones puede afectar a la polarización (Amblard y Deffuant, 2004; Deffuant et al., 2000; Franks et al., 2008; Hegselman y Krause, 2002; Macy et al., 2003).

Esta investigación comparte la visión de Acosta (2012) de que las redes sociales no son sino grupos que al organizarse en torno a unos valores y principios socialmente compartidos ponen de manifiesto una estructura polarizada entre unos y otros. 
Puede resultar extraño hablar de una polarización (normalmente entendida entre dos) en una campaña electoral en la que por primera vez en España había posibilidad de romper el bipartidismo, pero no lo es. El barómetro del CIS previo a la contienda del 26 de junio de 2016, de fecha 9 de junio, ya determinaba cuatro candidatos con posibilidades reales de obtener representación: Mariano Rajoy, Pedro Sánchez, Pablo Iglesias y Albert Rivera, como así fue. El 26J no se trataba, por tanto, de unas elecciones polarizadas de un partido contra otro, las redes no se orientaban en dos direcciones contrapuestas, sino en una combinación de cuatro contra cuatro.

Como explica Lozada (2004), la percepción estereotipada de los otros dificulta las posibilidades de dialogar, de llegar a acuerdos a partir del debate de ideas. En política esto es más evidente aún, ningún marco de referencia puede ser asumido como válido para todos, los valores dejan de tener significado colectivo.

Partimos de la investigación de Úbeda, F y Guerrero, E (2015) que va más allá de las definiciones de polarización política de Bramson et al. (2013); Fiorina \& Abrams (2008) y Mella (1999).

Ambos investigadores elaboraron una definición de polarización política en las redes en la que se tiene en cuenta la exposición selectiva que realizan los usuarios cuando se exponen a una cantidad de información no asimilable por el individuo y por la cual se conforman redes en torno a esas fuentes de información. Según afirman, dependiendo del grado de exposición, los usuarios serán más o menos impermeables a una información disonante con sus ideas.

Apoyan esta teoría estudios como el de Pew Research publicado en 2016 en el que se afirma que casi dos terceras partes de los usuarios de redes consideran estresante y frustrante hablar de política en redes con gente que mantiene opiniones diferentes (Dugan, M and Smith, A 2016).

En la política en las redes, tal y como afirma Sarlo (2011), la subjetividad de quien enuncia es importante. La mayoría de los estudios demuestran que en las redes, al no ser imprescindible la argumentación, el hecho de que los usuarios sigan una opción depende casi por completo del poder de persuasión del sujeto y no tanto del discurso.

Es importante tener en cuenta que en las redes sociales los usuarios son a la vez productores y comentadores activos de los contenidos que ponen en circulación (Jenkins, 2008, 2011; Scolari, 2013), de ahí que esta investigación compare los mensajes de los candidatos y de sus seguidores analizando la coincidencia, o no, de palabras en sus mensajes.

\section{Objetivos y metodología}

El objetivo de esta investigación es comparar cualitativamente los mensajes de los candidatos con los mensajes que reciben en sus comentarios, con el fin de poder aclarar si son los candidatos quienes polarizan o son los ciudadanos. Para ello, se analizará si las palabras más repetidas en los post y en los comentarios recibidos son las mismas o si difieren y, a la vez, se analizarán las palabras más repetidas en los 3 post que más comentarios han recibido de cada candidato, con el fin de determinar qué palabras pueden contribuir a generar mayor polarización en la sociedad.

Y también se procederá a investigar qué candidatos hablan más del resto de rivales, es decir, cuáles utilizan Facebook como forma de polarización con sus mensajes 
en comparación con otros y cuáles lo utilizan simplemente como método de información sin confrontación con el resto.

Para satisfacer estos objetivos hemos seleccionado el marco temporal que comprende la semana del 19 al 25 de junio de 2016, es decir, la última de campaña de las últimas elecciones generales celebradas en España. Y los cuatros candidatos elegidos son aquellos a los que el CIS de junio previo a las elecciones generales otorgaba mayor representatividad: Mariano Rajoy (PP), Pedro Sánchez (PSOE), Pablo Iglesias (Podemos) y Albert Rivera (Ciudadanos).

El corpus de la investigación es el siguiente:

\begin{tabular}{l|l|l}
\multicolumn{1}{c}{ Candidato } & \multicolumn{1}{c}{ Post } & Comentarios \\
\hline Mariano Rajoy & 19 & 4.042 \\
\hline Albert Rivera & 6 & 1.239 \\
\hline Alberto Garzón & 57 & 6.892 \\
\hline Pablo Iglesias & 13 & 20.076 \\
\hline Pedro Sánchez & 25 & 5.656 \\
\hline
\end{tabular}

Tabla 1. Elaboración propia

Para la recopilación del material de trabajo se usó Smétrica, una herramienta de mediciones en Facebook que tenía en seguimiento más de 16.000 páginas, de las que se recogían diariamente el número de fans, hablando de esto, publicaciones, comentarios, etc. Con cargo al proyecto "Las estrategias de campaña online de los partidos políticos españoles 2015-2016" de la Universidad Complutense de Madrid, dirigido por el Catedrático de Periodismo José Luis Dader y financiado por el Ministerio de Economía de España, se encargó a Smétrica que buscase todo lo relativo al muro de Facebook de los cuatro candidatos en la semana fijada para la investigación y se descargaron de la red social todos los post y todos los comentarios a estos post publicados en los perfiles individuales de los cuatro candidatos principales a las elecciones generales del 26 de junio de 2016: Mariano Rajoy, Albert Rivera, Pedro Sánchez y Pablo Iglesias.

Para el análisis del contenido de todos los textos se ha utilizado la herramienta AntConc en su versión 3.5.7 para Mac OSX (Anthony, 2018). Con esta herramienta de análisis de corpus para concordancia y análisis de texto se identificaron, en un primer momento, las palabras más repetidas en los post de cada uno de los candidatos.

A continuación, se escogieron los tres post de cada candidato con mayor número de comentarios sin tener en cuenta aquellos post en los que sólo se incluía un link ni los que se trataba de la retransmisión de vídeo en directo. Con la herramienta AntConc de nuevo se localizaron en el total de los comentarios de cada uno de los candidatos las menciones al resto, para poder descubrir con qué frecuencia la audiencia de un candidato menciona a cada uno de los otros. La búsqueda de las menciones de los candidatos se hizo por el primer apellido siendo Rajoy, Rivera y "Ribera" por si algún usuario cometiera el error ortográfico, Sánchez y "Sanchez" por el mismo motivo que el anterior y, por último, Iglesias. En todos los casos se estudió el significado de cada uno de los comentarios para asegurarse de que se trataba de una mención al candidato y no sufrir las consecuencias negativas en el análisis de posibles polisemias. En todos los casos la búsqueda se hizo sin tener en cuenta distinción entre mayúsculas y minúsculas. 


\section{Resultados}

Para el primero de los objetivos se analizaron las palabras más repetidas en los post de los cuatro candidatos estudiados y se compararon con las palabras más repetidas en los comentarios que recibieron Se buscaba determinar quién polariza más, los candidatos o sus seguidores.

Tabla 2. Análisis Rajoy

\begin{tabular}{|c|c|c|c|}
\hline \multicolumn{4}{|l|}{ Palabras más repetidas } \\
\hline \multirow[t]{10}{*}{ Post de Mariano Rajoy } & Partido & partido & \multirow{9}{*}{$\begin{array}{l}\text { Comentarios } \\
\text { a los post de } \\
\text { Mariano Rajoy }\end{array}$} \\
\hline & Voto & voto & \\
\hline & A favor & votar & \\
\hline & Ciudadanos & mejor & \\
\hline & Popular & suerte & \\
\hline & Extremistas & Rajoy & \\
\hline & España & España & \\
\hline & Radicales & señor & \\
\hline & Vota & Mariano & \\
\hline & Españoles & presidente & \\
\hline
\end{tabular}

Fuente: Elaboración propia

De Rajoy se analizaron 19 post y 4.042 comentarios. La conclusión tras el análisis de los datos de dicho candidato es que sus post polarizan más, utilizando palabras como extremistas y radicales, que los comentarios de sus seguidores, que utilizan un lenguaje menos agresivo en el que las palabras más repetidas son votar, suerte, presidente, España, etc... Todas las palabras utilizadas por sus seguidores son propias de cualquier contienda electoral y podrían ser utilizadas por cualquier candidato, mientras que las más utilizadas por el candidato ya presuponen una ideología y polarizan en mayor medida el discurso.

Tabla 3. Análisis Pedro Sánchez

\begin{tabular}{|c|c|c|c|}
\hline Palabras más repetidas & & & \\
\hline \multirow[t]{10}{*}{ Post de Pedro Sánchez } & cambio & Pedro & \multirow{9}{*}{$\begin{array}{l}\text { Comentarios } \\
\text { a los post de } \\
\text { Pedro Sánchez }\end{array}$} \\
\hline & PSOE & PSOE & \\
\hline & Gobierno & Voto & \\
\hline & país & partido & \\
\hline & nuestro & Pablo & \\
\hline & derechos & cambio & \\
\hline & España & España & \\
\hline & Rajoy & unidos & \\
\hline & partido & votar & \\
\hline & socialista & presidente & \\
\hline
\end{tabular}

Fuente: Elaboración propia 
De Pedro Sánchez se analizaron 25 post y un total de 5.656 comentarios recibidos. Entre los datos del candidato Pedro Sánchez, sin embargo, no se da esta circunstancia. Sus palabras más repetidas y las de sus seguidores son muy parecidas en cuanto al nivel de polarización que generan. Los vocablos genéricos aluden al cambio o a gobernar, no tienen una carga negativa y son perfectamente intercambiables con otros candidatos opositores, pero se da una circunstancia muy curiosa. Los post del candidato incluyen mención directa a Mariano Rajoy y, sin embargo, sus seguidores en quien personalizan es en Pablo Iglesias. Parece que no hay coincidencia entre quién considera Pedro Sánchez su rival más importante y la percepción que tienen sus seguidores.

También es importante destacar que entre las palabras más repetidas por el candidato hay alguna que introduce un matiz más social, "derechos", una inclusión que permite utilizar también este análisis del discurso para determinar los conceptos clave de cada partido.

Tabla 4. Análisis Pablo Iglesias

\begin{tabular}{|c|c|c|c|}
\hline \multicolumn{4}{|l|}{ Palabras más repetidas } \\
\hline \multirow[t]{10}{*}{ Post de Pablo Iglesias } & campaña & unidos & \multirow{10}{*}{$\begin{array}{l}\text { Comentarios } \\
\text { a los post de } \\
\text { Pablo Iglesias }\end{array}$} \\
\hline & esto & domingo & \\
\hline & cierre & Pablo & \\
\hline & Europa & ganar & \\
\hline & directo & gracias & \\
\hline & abrazos & cambio & \\
\hline & domingo & España & \\
\hline & abuela & gente & \\
\hline & día & votar & \\
\hline & acercarme & presidente & \\
\hline
\end{tabular}

Fuente: Elaboración propia

De Pablo Iglesias se analizaron 13 post y 20.076 comentarios. Es el candidato que más comentario recibe de los cuatro estudiados. En su caso llama la atención que el candidato introduce palabras mucho más emocionales que el resto de los candidatos analizados entre sus vocablos más repetidos: abrazos, abuela, acercarme.... Su nivel de polarización es muy bajo y coincide con el de sus seguidores a través de sus comentarios.

Se da un dato curioso, entre las palabras más repetidas por sus seguidores no figura otro candidato, sino el propio Pablo Iglesias, lo que pone de manifiesto el total personalismo de esta candidatura a pesar de ser una candidatura asamblearia. El seguimiento de su líder es total. 
Tabla 5. Análisis Albert Rivera

\begin{tabular}{|c|c|}
\hline Palabras más repetidas & \\
\hline Comentarios de Albert Rivera & Albert \\
\hline & Rajoy \\
\hline & ahora \\
\hline & España \\
\hline & voto \\
\hline & PSOE \\
\hline & partido \\
\hline & ciudadanos \\
\hline & votar \\
\hline & Rivera \\
\hline
\end{tabular}

Fuente: Elaboración propia

Para el último de los candidatos, Albert Rivera, no se pudo elaborar una lista de las diez palabras con más frecuencia en sus post debido a la escasez de contenido. En el perfil de este candidato sólo se publicaron 6 post con una extensión de texto muy breve, por lo que no se pudo identificar un grupo de palabras relevante que se repitieran con una frecuencia representativa superior a las demás. Estos post reunieron 1.239 comentarios.

A pesar de la carencia anteriormente mencionada sí que se reconoció el término "españoles" con una frecuencia superior a las demás. Se trata claramente de una estrategia importante, ya que Ciudadanos es un partido surgido en Cataluña, donde hay un fuerte problema con el nacionalismo y el partido de Albert Rivera se quiere presentar como el partido de la unidad de todos los españoles. De ahí que repitan hasta la saciedad en sus escasos post esta palabra y sea la única que se coloca con datos estadísticos significativos entre las más repetidas.

La palabra "españoles", además, le sirve a Ciudadanos para polarizar el debate con el PP, su rival a batir, ya que muchos seguidores del Partido Popular achacaban precisamente a su líder falta de defensa de la unidad de España, algo que, a juzgar por las palabras más repetidas de Rajoy no es cierto, ya que entre sus diez aparecen tanto españoles como España.

En el análisis de las palabras de sus seguidores sí se pueden determinar las más repetidas e igual que ocurre con el candidato de Podemos, los seguidores de Ciudadanos colocan las palabras Albert y Rivera entre las más mencionadas. Se trata, pues, de una candidatura también muy personalista.

Y también polarizan mucho contra la figura de Mariano Rajoy, que aparece entre las palabras más repetidas porque es a quién los seguidores de Ciudadanos sitúan como el candidato a derrotar en estos comicios.

Se comprueba, por tanto, que en el análisis de quién polariza más, los candidatos o los seguidores, el líder del PP, Mariano Rajoy, es el único que aparece por encima del nivel de polarización de sus seguidores. Esto puede significar que Rajoy es un candidato más agresivo que el resto o que sus seguidores lo son menos que los de sus contrincantes. Cualquiera de las dos interpretaciones es posible y, sin más datos, no se puede determinar cuál es cierta. 
El resto de los candidatos, Pedro Sánchez, Pablo Iglesias y Albert Rivera polarizan en el mismo nivel que quienes hacen los comentarios a sus post. Estos datos sobre quién polariza más, los candidatos o sus seguidores, recordamos que se lograron analizando todos los post y comentarios especificados en la Tabla 1.

Pero esta investigación pretendía ir más allá e intentar determinar si había una lista de palabras clave que despertaran la participación masiva de los seguidores políticos de los cuatro líderes y que, de facto, llevaran a una polarización de los comentarios.

Para este segundo objetivo se buscaron los tres post de los cuatro candidatos que más seguidores tuvieron y se analizaron cualitativamente sus mensajes. No se han tenido en cuenta aquellos post que consistían en la difusión de links ni los vídeos en directo. La recopilación recoge post que son en su totalidad imágenes.

Tabla 6. Elaboración propia

\begin{tabular}{|c|c|c|c|c|c|}
\hline Fecha publicación & Nombre autor & Mensaje & Likes & $\begin{array}{c}\text { Veces } \\
\text { compartido }\end{array}$ & Comentarios \\
\hline $\begin{array}{l}2016-06- \\
25 \mathrm{~T} 10: 52: 21+0000\end{array}$ & $\begin{array}{l}\text { Mariano Rajoy } \\
\text { Brey }\end{array}$ & $\begin{array}{l}\text { \#JornadaDeReflexión, un poco de depor- } \\
\text { te con mi perro Rico antes de pasar un } \\
\text { agradable día en familia. \#FelizSábado }\end{array}$ & 7003,00 & 447,00 & 830,00 \\
\hline $\begin{array}{l}\text { 2016-06- } \\
\text { 22T21:28:04+0000 }\end{array}$ & $\begin{array}{l}\text { Mariano Rajoy } \\
\text { Brey }\end{array}$ & $\begin{array}{l}\text { Me he divertido mucho en El Hormigue- } \\
\text { ro con Pablo Motos, su equipo y Trancas } \\
\text { y Barrancas. Entretenimiento, risas y } \\
\text { buena compañía para hacer deporte. Un } \\
\text { placer. } \\
\text { Os invito a ver el programa completo: } \\
\text { http://ow.ly/gepj301xr9A \#RajoyEH }\end{array}$ & 9283,00 & 1389,00 & 602,00 \\
\hline $\begin{array}{l}\text { 2016-06- } \\
24 \mathrm{~T} 21: 58: 02+0000\end{array}$ & $\begin{array}{l}\text { Mariano Rajoy } \\
\text { Brey }\end{array}$ & $\begin{array}{l}\text { Militantes, simpatizantes, apoderados, } \\
\text { interventores, técnicos, voluntarios... } \\
\text { igracias! Orgulloso de vuestro trabajo } \\
\text { y empuje. El domingo todos a votar al } \\
\text { Partido Popular. Si quieres un país unido } \\
\text { y no una España gobernada por los } \\
\text { radicales, \#piénsatelo, no te arriesgues y } \\
\text { apuesta por lo seguro; concentra el voto } \\
\text { por una \#EspañaAFavor, \#VotaPP. }\end{array}$ & 5527,00 & 995,00 & 330,00 \\
\hline $\begin{array}{l}\text { 2016-06- } \\
20 \mathrm{~T} 18: 47: 02+0000\end{array}$ & Albert Rivera & $\begin{array}{l}\text { Si ponemos en marcha un nuevo } \\
\text { gobierno, en } 100 \text { días haremos cambios } \\
\text { importantes para la mayoría de } \\
\text { españoles. \#26J }\end{array}$ & 6709,00 & 1964,00 & 358,00 \\
\hline $\begin{array}{l}\text { 2016-06- } \\
\text { 24T12:36:11+0000 }\end{array}$ & Albert Rivera & $\begin{array}{l}\text { Lamento la decisión de \#Reino Unido de } \\
\text { abandonar el proyecto común. El miedo } \\
\text { y el rencor venció a la oportunidad. } \\
\text { Ahora toca más Europa }\end{array}$ & 3796,00 & 256,00 & 253,00 \\
\hline $\begin{array}{l}\text { 2016-06- } \\
\text { 19T21:43:15+0000 }\end{array}$ & Albert Rivera & $\begin{array}{l}\text { Orgullosos de ser nuevos, de centro, de } \\
\text { querer cambio, de darnos la mano, de } \\
\text { ser inconformistas, de ser ciudadanos } \\
\text { \#MadridNaranja }\end{array}$ & 3929,00 & 267,00 & 249,00 \\
\hline $\begin{array}{l}\text { 2016-06- } \\
\text { 20T20:05:05+0000 }\end{array}$ & Pablo Iglesias & $\begin{array}{l}\text { Estos } 177 \text { economistas han lanzado un } \\
\text { manifiesto pidiendo el fin de las políticas } \\
\text { de austeridad. Queremos agradecerles su } \\
\text { compromiso. }\end{array}$ & 27224,00 & 7962,00 & 865,00 \\
\hline
\end{tabular}




\begin{tabular}{|c|c|c|c|c|c|}
\hline $\begin{array}{l}\text { 2016-06- } \\
23 \mathrm{~T} 10: 18: 51+0000\end{array}$ & Pablo Iglesias & $\begin{array}{l}\text { Este viernes no solo termina una } \\
\text { campaña, empieza el camino hacia un } \\
\text { futuro mejor. No faltéis }\end{array}$ & 6209,00 & 986,00 & 433,00 \\
\hline $\begin{array}{l}\text { 2016-06- } \\
22 \mathrm{~T} 16: 06: 20+0000\end{array}$ & Pablo Iglesias & $\begin{array}{l}\text { Gracias, Ángela. Todo esto tiene sentido } \\
\text { por } 1 @ \text { s que habéis luchado tanto. Tu } \\
\text { nieta estará orgullosa. }\end{array}$ & 11767,00 & 1051,00 & 338,00 \\
\hline $\begin{array}{l}\text { 2016-06- } \\
\text { 25T13:04:28+0000 }\end{array}$ & $\begin{array}{l}\text { Pedro Sánchez } \\
\text { Pérez-Castejón }\end{array}$ & $\begin{array}{l}\text { \#JornadaDeReflexión disfrutando en } \\
\text { casa con la familia y con los amigos de } \\
\text { siempre. ¡¡Buen sábado a todos!! }\end{array}$ & 4806,00 & 431,00 & 567,00 \\
\hline $\begin{array}{l}\text { 2016-06- } \\
24 \mathrm{~T} 12: 13: 16+0000\end{array}$ & $\begin{array}{l}\text { Pedro Sánchez } \\
\text { Pérez-Castejón }\end{array}$ & $\begin{array}{l}\text { ¡Buenas tardes desde Madrid! } \\
\text { En las últimas horas de la campaña } \\
\text { electoral, va a haber muchos que apelen } \\
\text { al voto de miedo y al de la ruptura, } \\
\text { y quiero recordaros que eso solo trae } \\
\text { bloqueo y frustración en la política y en } \\
\text { la vida social española. } \\
\text { Después de cuatro años de corrupción, } \\
\text { recortes y mentiras, España necesita } \\
\text { políticos limpios, políticos que no } \\
\text { escondan nada. Rajoy es un político } \\
\text { en B, tiene un programa político en } \\
\text { B, una financiación en B... pero hay } \\
\text { muchas cosas que no se pueden tener } \\
\text { en B: la diligencia, la honestidad y el } \\
\text { compromiso. Porque el compromiso, se } \\
\text { tiene o no se tiene; Rajoy no lo tiene y el } \\
\text { \#PSOE sí lo tiene. } \\
\text { Además, ya sabemos que en campaña } \\
\text { electoral Mariano Rajoy aporta sesudas } \\
\text { reflexiones políticas. Después de cuatro } \\
\text { años ha reconocido haber descubierto } \\
\text { que en España hay españoles, y lo que } \\
\text { los españoles queremos saber es qué hay } \\
\text { en Panamá y Suiza. } \\
\text { Por otro lado, en estos dos años de } \\
\text { existencia de su partido, Iglesias no ha } \\
\text { hecho otra cosa que cambiar. Cambia } \\
\text { tanto, que el } 2 \text { y } 4 \text { de marzo acabó } \\
\text { votando con el PP en contra de un } \\
\text { presidente socialista. } \\
\text { Las elecciones del próximo domingo son } \\
\text { muy importantes, son trascendentales } \\
\text { porque España, junto al resto de Europa, } \\
\text { está viviendo dos tensiones: rupturismo } \\
\text { e inmovilismo. } \\
\text { Lo que ha ocurrido en el Reino Unido } \\
\text { es gravísimo, es un golpe muy duro para } \\
\text { todos los que somos europeístas, para el } \\
\text { proyecto común que representa la Unión } \\
\text { Europea, pero no es un golpe definitivo. }\end{array}$ & 3304,00 & 831,00 & 338,00 \\
\hline
\end{tabular}




\begin{tabular}{|c|c|c|c|c|c|}
\hline & & $\begin{array}{l}\text { Los europeístas debemos reaccionar } \\
\text { y reivindicar a los grandes líderes } \\
\text { socialistas que tanto hicieron por la } \\
\text { clase media y trabajadora, como hizo } \\
\text { Felipe González cuando era presidente } \\
\text { del gobierno. Lo que ha ocurrido en el } \\
\text { Reino Unido es la confluencia entre una } \\
\text { derecha irresponsable y el populismo, } \\
\text { y yo no quiero eso para España. La } \\
\text { solución para nuestro país es un partido } \\
\text { socialista fuerte, ganador, que apueste } \\
\text { por la reforma y el progreso, que es lo } \\
\text { que representa la socialdemocracia. } \\
\text { España tiene un gran partido socialista, } \\
\text { es el cambio que suma derechos, } \\
\text { oportunidades, libertades y tolerancia. } \\
\text { Os pido que el \#26J vayáis a votar con } \\
\text { ilusión y emoción por un cambio seguro } \\
\text { y limpio. } \\
\text { Vamos a ser protagonistas del cambio } \\
\text { político, construyendo una unión } \\
\text { de valores solidarios, democráticos, } \\
\text { fundamentados en la igualdad y la } \\
\text { libertad. } \\
\text { Os pido que no votéis con miedo ni } \\
\text { rencor, y que apostéis por una España } \\
\text { mejor en una Europa mejor, que votéis } \\
\text { con ilusión y esperanza para hacer } \\
\text { avanzar a nuestro país. } \\
\text { \#VotaPSOE \#UnSíAEuropa \#26J } \\
\text { \#UnSíPorElCambio }\end{array}$ & & & \\
\hline $\begin{array}{l}\text { 2016-06- } \\
23 \mathrm{~T} 20: 14: 59+0000\end{array}$ & $\begin{array}{l}\text { Pedro Sánchez } \\
\text { Pérez-Castejón }\end{array}$ & $\begin{array}{l}\text { ¡Buenas y calurosas noches! } \\
\text { Os voy a pedir aquí en Murcia que os } \\
\text { mojéis y votéis SÍ al cambio. Hoy tengo } \\
\text { el inmenso honor de ser el candidato } \\
\text { a la presidencia del gobierno por el } \\
\text { partido que más quiero, el PSOE. Y } \\
\text { lo hago con orgullo y con memoria. } \\
\text { Con la memoria del legado de nuestros } \\
\text { abuelos y abuelas, a los que damos las } \\
\text { gracias. El legado de un país que sufrió } \\
\text { durante años crisis y guerras pero que } \\
\text { nunca se rindió hasta conseguir derechos } \\
\text { y libertades. Nosotros no vamos a } \\
\text { despreciar su legado como hacen otros. } \\
\text { También os pido un sí por el cambio } \\
\text { por el presente, el presente de nuestros } \\
\text { padres y madres y por el futuro, el de } \\
\text { nuestros hijos e hijas. } \\
\text { Mirad, nunca con el apoyo de la derecha } \\
\text { hemos conseguido nada. Cuando España } \\
\text { ha necesitado cambio, hemos llenado } \\
\text { las urnas de votos socialistas. Volvamos } \\
\text { a hacerlo. }\end{array}$ & 2849,00 & 553,00 & 278,00 \\
\hline
\end{tabular}




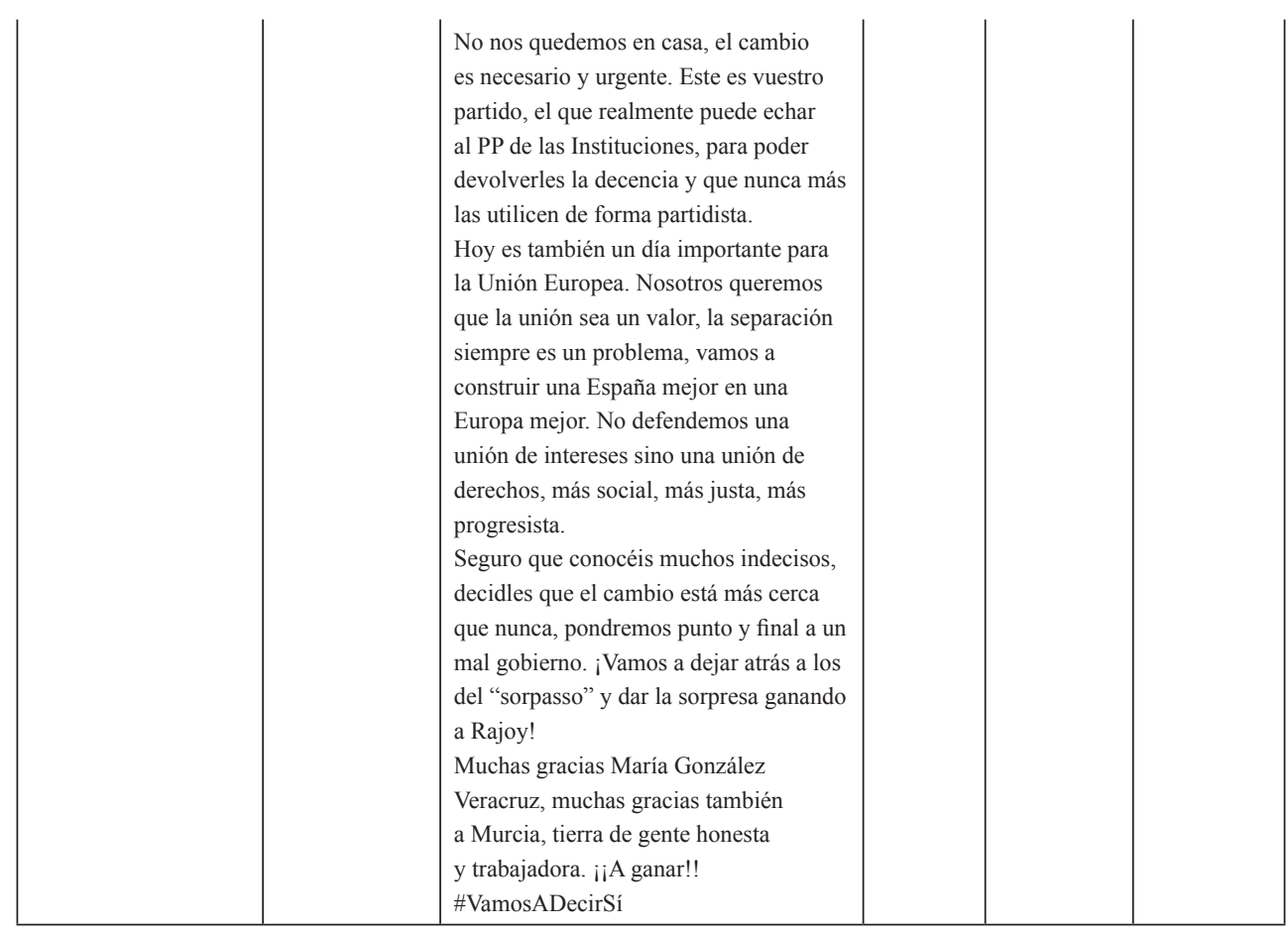

Tras seleccionar los post, éste es el descriptivo:

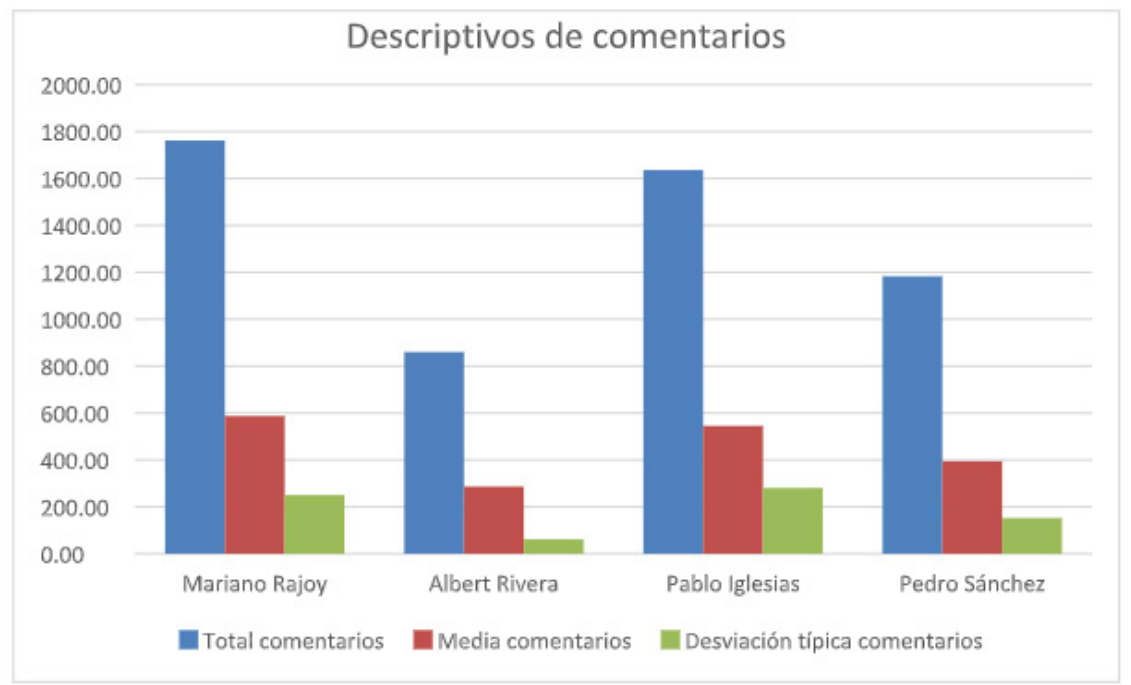

Gráfico 1. Elaboración propia

Analizando el discurso de los post que más seguidores lograron no cabe dudas: Cada candidato utilizó las palabras que sabía que activarían la palanca de la participación entre sus fieles en las redes. En el caso de Rajoy, los post con más seguimiento fueron curiosamente los personales, los que alejaban la imagen de un presidente distante. Las palabras clave utilizadas fueron deporte, risas, entretenimiento, agra- 
dable... Pero la idea política fuerza fue la de transmitir que los españoles no debían arriesgar para que España no fuera gobernada por los radicales. Precisamente la palabra "radicales" era una de las que aparecía entre las diez más mencionadas por el candidato en todos los post analizados durante la semana del estudio.

Los seguidores del PP, según la encuesta de Metroscopia del 25 de mayo de 2016, son "envejecidos, conservadores y contrarios al multipartidismo", por lo que la amenaza de un gobierno multicolor integrado por radicales era un arma para movilizarles no solo a las redes, sino también a las urnas. Como ya apuntaron Abejón y Mayoral (2017), la técnica de la repetición es una de las técnicas de persuasión más utilizadas por los candidatos y más efectivas.

En el caso de Pedro Sánchez, hay que destacar que la extensión de sus post es inmensa en comparación con la del resto de los candidatos. Sus intervenciones en Facebook son casi minidiscursos de campaña y las palabras clave son cambio, derechos, populismo y derecha irresponsable. Muchas de ellas también coinciden con las diez palabras más repetidas por el candidato en el cómputo general de la semana (Tabla 1).

Pedro Sánchez polariza mucho en los post con mayor número de seguidores. Claramente identifica a Rajoy y al PP con la corrupción, calificándole como un político en B, y contrapone la idea de un político limpio que representa él. Y también polariza identificando a Pablo Iglesias con el populismo.

Frente a todo esto, Pedro Sánchez presenta al PSOE y su candidatura como el necesario cambio y otorga a sus seguidores un papel muy importante, el de protagonistas de ese cambio.

Por su parte, Pablo Iglesias es el candidato que menos de sus palabras más repetidas en la Tabla 1 utilizó en los tres post que más seguidores le hicieron tener.

Las palabras clave de sus post que más seguidores consiguieron son "futuro mejor", "austeridad" y "compromiso" y no todas están entre sus palabras más repetidas en Facebook.

Curiosamente, los post más seguidos de Pablo Iglesias hablan de economía. El candidato de Podemos quiere romper esa imagen difundida de que su partido no sabría cómo gobernar, quiere presentarse como un partido solvente en materia económica y, a la vez, explota la debilidad de su adversario: habla de austeridad porque la gente ha llegado a aborrecer esa palabra y a quién la ha puesto en sus vidas, el PP.

Por su parte, Albert Rivera enfatizó en estos tres post con más seguidores los conceptos clave de su campaña, "nuevos" y "de centro". Con estas ideas y con la palabra "cambio" fueron con las que lograron su mayor número de seguidores.

Finalmente, esta investigación abordó el tema de la polarización desde el ángulo de la mención al rival. Si hay algo que polariza el debate en política y que queríamos analizar es la búsqueda del enfrentamiento con el contrincante. Por ello, hemos analizado las menciones de cada candidato a sus rivales para ver quién confrontó más durante la campaña del 26J.

Las menciones en los post de los candidatos quedaban ya reflejadas en las Tablas 2, 3, 4 y 5. En ellas vemos como entre las palabras más utilizadas por Rajoy está Ciudadanos y radicales, que era el adjetivo para referirse a Podemos; entre las más utilizadas por Pedro Sánchez está directamente Rajoy, entre las de Albert Rivera está también Rajoy y el único candidato que en sus post no polariza buscando a un rival es Pablo Iglesias, que en sus post no menciona a ningún rival.

Sin embargo, como entendemos que mantener el Facebook de los candidatos sin muestras de agresividad o conflicto es una lección que los expertos en redes de cam- 
pañas políticas utilizan hemos analizado los comentarios que recibieron esos post para ver si los seguidores polarizaban el debate político atacando a los contrincantes de sus líderes. Estos son los resultados:

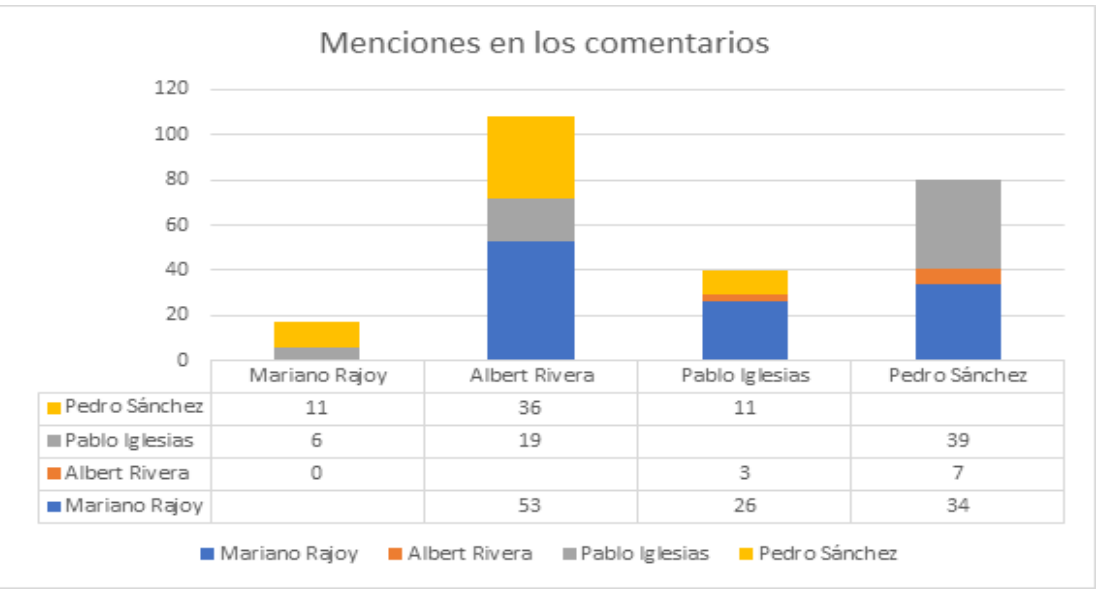

Gráfico 2. Elaboración propia

Según estos resultados, los candidatos cuyos seguidores más polarizaron el debate político en Facebook durante la campaña electoral del 26J fueron los de Albert Rivera y los de Pedro Sánchez, seguidos de los de Pablo Iglesias. En último lugar, los seguidores de Mariano Rajoy apenas mencionaron a sus rivales y cuando lo hicieron fue ignorando a Ciudadanos (0 alusiones) y centrándose en los candidatos de PSOE y Podemos.

Analizando el gráfico se puede determinar que, sin embargo, el rival del que más hablaron los seguidores de Ciudadanos fue de Mariano Rajoy. Los seguidores de Pedro Sánchez se repartieron casi por igual entre los mensajes contra Pablo Iglesias y contra Rajoy y los de Pablo Iglesias, sin embargo, centraron sus comentarios principalmente en Rajoy.

\section{Discusión y conclusiones}

Hay autores como Barberá (2015) que han determinado que un mayor uso de las redes sociales (Twitter, en este caso) medido con la variable exposure to diversity (exposición a la diversidad a través del uso de Twitter) está asociado a una menor polarización política.

La investigación de Barberá (2015) se realizó de forma paralela en España, Alemania, y Estados Unidos y es coincidente con la de Boxel et al (2017), que sugiere que es difícil sostener la hipótesis de que internet es el medio que genera mayor polarización. La estrategia de Boxel y otros fue usar datos de encuestas para calcular un índice de polarización y analizar cómo este índice ha cambiado en los recientes años para individuos de diferentes edades. Sus datos muestran que la tasa de uso de internet de los ancianos para seguir las noticias es mucho menor a la tasa de los individuos de media edad y, sin embargo, éstos están mucho más polarizados políticamente que los jóvenes, que sí son usuarios habituales de la red. 
En el lado opuesto hay múltiples estudios que hablan de que la red no sólo polariza, sino que va un paso más allá y favorece, incluso, lo que numerosos estudiosos denominan la descortesía (Kaul y Cordisco, 2014). Este fenómeno se da en internet debido a que los usuarios carecen de las restricciones coercitivas que rigen en la sociedad off-line. Siempre es más difícil decir a la cara cosas que el anonimato favorece.

La presente investigación permite demostrar que los candidatos a la presidencia del Gobierno en España buscaron polarizar a la sociedad desde sus cuentas de Facebook durante la última campaña electoral, la celebrada para las elecciones del 26 de junio de 2016.

Todos, a excepción de Pablo Iglesias, aludieron a sus oponentes con nombres de forma masiva en sus post, lo que polariza a quienes les siguen tal y como sostiene Van Dijk (2003): "la polarización es una estrategia discursiva en la que se pone en marcha el mecanismo global de auto-presentación positiva de unos frente a la presentación negativa de los otros".

Entre los comentarios a los post, la investigación demuestra que los seguidores que más polarizaron fueron los de Albert Rivera, seguidos de los de Pedro Sánchez, mientras los que menos aludieron a sus rivales para generar debate fueron los del PP.

Respecto a si hay palabras que generan más polarización que otras la conclusión no es clara, ya que las más utilizadas por los cuatro candidatos en sus post con más seguidores no coinciden. Lo que sí queda demostrado, en consonancia con lo expuesto por Abejón y Mayoral (2017) es que la técnica de persuasión de la repetición del mensaje es muy utilizada por los candidatos, ya que la mayoría de los vocablos que aparecen entre los más repetidos en todos sus post también están en los tres con más seguidores. Nuevamente aquí la excepción es Pablo Iglesias.

\section{Bibliografía}

Abejón-Mendoza, P. \& Mayoral-Sánchez, J., 2017. Persuasión a través de Facebook de los candidatos en las elecciones generales de 2016 en España. El Profesional de la Información, 26(5), p. 928.

Acosta, V., \& Lucia, G. (2017). Gestión de pasiones y polarización en las redes sociales. Un análisis del aplicativo Grupos en Facebook. Discurso \& Sociedad, 2012, Vol. 6 (4), pp. 684-719.

Albarello, F. (2013). Carlos Scolari. Narrativas transmedia. Cuando todos los medios cuentan. Austral Comunicación, 2(2), pp. 247-249.

Amblard, F., \& Deffuant, G. (2004). The role of network topology on extremism propagation with the relative agreement opinion dynamics. Physica A: Statistical Mechanics and its Applications, 343, pp. 725-738.

Anthony, L. (2018). AntConc (Version 3.5.7) [Computer Software]. Tokyo, Japan: Waseda University. http://www.laurenceanthony.net/software

Barberá, P. (2014). How social media reduces mass political polarization. Evidence from Germany, Spain, and the US. Job Market Paper, New York University, p. 46.

Boxell, L., Gentzkow, M., \& Shapiro, J. M. (2017). Is the internet causing political polarization? Evidence from demographics (No. w23258). National Bureau of Economic Research. 
Díaz, J. B. (2013). Twitter y la polarización del debate político: análisis del caso\# objetivodeguindos y\# aznara3/Twitter and polarization of political disscusion: the case\# objetivodeguindos y\# aznara3. Historia y Comunicación Social, 18, pp. 499-509.

Duggan, M., \& Smith, A. (2016). The tone of social media discussions around politics. Pew Research Center http://www. pewinternet. org/2016/10/25/the-tone-of-social-media-discussions-around-politics.

Franks, D. W., Noble, J., Kaufmann, P., \& Stagl, S. (2008). Extremism propagation in social networks with hubs. Adaptive Behavior, 16(4), pp. 264-274.

Hegselmann, R., \& Krause, U. (2002). Opinion dynamics and bounded confidence models, analysis, and simulation. Journal of artificial societies and social simulation, 5(3).

Jenkins, H., \& Lazcano, P. H. (2008). Convergence culture: La cultura de la convergencia de los medios de comunicación (p. 301). Editorial Paidós.

- (2011). Transmedia Storytelling 202: Further Reflections, en Confessions of an Aca-Fan. https://bit.ly/2LtW1fS.

Jiang, Y. (2014). 'Reversed agenda-setting effects' in China Case studies of Weibo trending topics and the effects on state-owned media in China. Journal of International Communication, 20(2), pp. 168-183.

de Marlangeon, S. B. K., \& Cordisco, A. (2014). La descortesía verbal en el contexto político-ideológico de las redes sociales. Revista de Filología de la Universidad de La Laguna, (32), pp. 145-162.

Lozada, M. (2004). El otro es el enemigo: imaginarios sociales y polarización. Revista Venezolana de Economía y Ciencias Sociales, 10(2).

Macy, M. W., Kitts, J. A., Flache, A., \& Benard, S. (2003). Polarization in dynamic networks: A Hopfield model of emergent structure. Dynamic social network modeling and analysis, pp. 162-173.

Martín-Baró, I. (1983). Polarización social en el Salvador. ECA Estudios Centroamericanos (1983), vol. 38, no. 412, pp. 129-142.

Raigada, J. L. P. (2002). Epistemología, metodología y técnicas del análisis de contenido. Estudios de sociolingüística, 3(1), pp. 1-42.

Kumar, S., Hamilton, W. L., Leskovec, J., \& Jurafsky, D. (2018, April). Community interaction and conflict on the web. In Proceedings of the 2018 World Wide Web Conference on World Wide Web (pp. 933-943). International World Wide Web Conferences Steering Committee.

Vasco, S. S. P., Spura, F. Ú., \& Salgado, E. G. Polarización política: análisis de la relación interpartidista en Twitter. Acercamiento al caso comparado España-Ecuador.

van Dijk, T. A. (1999). Ideology: A Multidisciplinary Approach. Gedisa.

- (2003). Ideología y discurso: una introducción multidisciplinaria. Ariel. 